\title{
Identification and analysis of long non- coding RNAs in response to H5N1 influenza viruses in duck (Anas platyrhynchos)
}

\author{
Chang Lu', Yanling Xing ${ }^{1}$, Han Cai ${ }^{1}$, Yirong Shi ${ }^{1}$, Jinhua Liu² and Yinhua Huang ${ }^{1 *}$
}

\begin{abstract}
Background: Long non-coding RNAs (IncRNAs) are important component of mammalian genomes, where their numbers are even larger than that of protein-coding genes. For example, human (Homo sapiens) $(96,308$ vs. 20,376) and mouse (Mus musculus) $(87,774$ vs. 22,630$)$ have more IncRNA genes than protein-coding genes in the NONCODEv5 database. Recently, mammalian IncRNAs were reported to play critical roles in immune response to influenza A virus infections. Such observation inspired us to identify IncRNAs related to immune response to influenza A virus in duck, which is the most important natural host of influenza A viruses.

Results: We explored features of 62,447 IncRNAs from human, mouse, chicken, zebrafish and elegans, and developed a pipeline to identify IncRNAs using the identified features with transcriptomic data. We then collected 151,970 assembled transcripts from RNA-Seq data of 21 individuals from three tissues and annotated 4094 duck IncRNAs. Comparing to duck protein-coding transcripts, we found that 4094 IncRNAs had smaller number of exons (2.4 vs. 10.2) and longer length of transcripts (1903.0 bp vs. 1686.9 bp) on average. Among them, 3586 (87.6\%) IncRNAs located in intergenic regions and 619 IncRNAs showed differential expression in ducks infected by H5N1 virus when compared to control individuals. 58 IncRNAs were involved into two co-expressional modules related to anti-influenza A virus immune response. Moreover, we confirmed that eight IncRNAs showed remarkably differential expression both in vivo (duck individuals) and in vitro (duck embryo fibroblast cells, DEF cells) after infected with H5N1 viruses, implying they might play important roles in response to influenza A virus infection.

Conclusions: This study presented an example to annotate IncRNA in new species based on model species using transcriptome data. These data and analysis provide information for duck IncRNAs' function in immune response to influenza A virus.
\end{abstract}

Keywords: Duck, LncRNAs, Avian H5N1 influenza virus, Immune response

\section{Background}

Transcriptome analysis has revealed that $80 \%$ of eukaryotic (such as human [1] and mouse [2]) genomes are transcribed, but $1-2 \%$ of the genome encodes proteins [3], suggesting that a large number of transcripts were non-coding RNAs (ncRNAs). Among ncRNAs, lncRNAs distribute extensively in animal and plant genomes. LncRNAs are transcripts being longer than 200 nucleotides, which were previously reported not to encode functional proteins [4]. However, recent studies

\footnotetext{
* Correspondence: cauhyh@cau.edu.cn

'State Key Laboratory of Agrobiotechnology, China Agricultural University, Beijing 100193, China

Full list of author information is available at the end of the article
}

found that some lncRNAs (such as DWORF [5] and SPAR [6]) encoded peptides and played important roles in the myocardial contraction and muscle regeneration, respectively. Such observation updated our knowledge about lncRNAs, which may not only affect transcription of gene expression with non-coding transcripts [7, 8], but also encode polypeptides to negatively regulate protein activation with its peptide [9].

Duck is one of the important economical waterfowl and the natural reservoir of all influenza A viruses harboring 18 hemagglutinin (HA) and 11 neuraminidase (NA) subtypes [10], with the exception of the H13, H16, H17 and H18 subtypes [11]. Compared to chicken being destroyed by influenza A virus (such as H5N1 viruses), 
ducks are tolerant to most subtypes of $\mathrm{H} 5 \mathrm{~N} 1$ viruses [12]. RIG-I (Retinoic Acid Inducible Gene I), one of the important intracellular viral RNA detector, is absent in chicken but play a critical role in immune response to H5N1 virus infections, partly contributing to the stark difference in influenza pathology between ducks and chickens [13]. Our previous studies indicated that duck might diversify their immune coding genes, such as $\beta$-defensin and butyrophilin-like genes, to optimize their immune response to influenza A virus [14]. Recently, lncRNAs were found to modulate influenza viral infection in mammals. For example, $N R A V$ inhibits the transcription of interferon-stimulated genes (ISGs) by affecting their histone modification, thus promotes replication of influenza A virus in human and mouse [15]. Similarly, mouse VIN promotes the replication of influenza viruses [16]. However, lncRNAs were only characterized on few birds (i.e. chicken and zebra finch) [17, 18] and their functions in anti-influenza immune response in birds are not clear.

Here, we developed a systematical pipeline for lncRNA identification and annotated 4094 duck lncRNAs using brain, lung and spleen transcriptomes of control individuals and ones that were infected with a highly pathogenic (A/duck/Hubei/49/05) or a weakly pathogenic (A/goose/ Hubei/65/05) H5N1 virus (DK/49 or GS/65). We compared the genomic structure and expression pattern of duck lncRNAs to their protein-coding genes and identified the differentially expressed lncRNAs in H5N1 virus infected duck and control individuals. We further identified lncRNAs and protein-coding genes co-expressional modules. Moreover, we qualified transcripts of eight lncRNAs by qRT-PCR and found these lncRNAs showing remarkably differential expression in both $\mathrm{H} 5 \mathrm{~N} 1$ virus infected duck and DEF cells. The findings offer new information for functional studies of duck lncRNAs, especially in immune response to influenza A viruses.

\section{Results}

Development a pipeline for the identification of IncRNA

A suitable pipeline for the identification of duck lncRNA was developed as the following: (1) explore features of 62,447 lncRNAs of five model organisms (human, Homo sapiens; mouse, Mus musculus; chicken, Gallus gallus; zebrafish, Danio rerio; elegans, Caenorhabditis elegans) from the GENCODE and NONCODE databases. We found that most lncRNA transcripts are $>200$ nucleotides (nt) in length, have more than one exon, and the length of open reading frame $(\mathrm{ORF})$ are $<100$ amino acids (AA) (Additional file 1: Figure S1); (2) build a strict platform to identify putative lncRNA transcripts larger than $200 \mathrm{nt}$ and having ORFs shorter than 100 AA according to the features of lncRNA in five model organisms (Fig. 1) [19]; (3) the putative lncRNA is aligned to known protein sequences using
BlastX ( $E$ value $<1 \times 10^{-3}$ ) and filtered with the protein database of KEGG (Kyoto Encyclopedia of Genes and Genomes), Swiss-Prot (Swiss-Protein database) and the NR data set from NCBI, as well as duck reference gene set, using the Cuffcompare program from the Cufflinks package $[14,17,20]$; (4) assess the protein-coding potential of putative lncRNAs using the Coding Potential Calculator (CPC) [21]; (5) filter putative IncRNA with only one exon.

\section{Genome-wide annotation of duck IncRNAs}

A total of 2,592,396,390 clean reads from 21 available duck transcriptomes of three tissues (brain, spleen and lung) with or without infection by a highly pathogenic (A/duck/Hubei/49/05) or a weakly pathogenic (A/goose/ Hubei/65/05) H5N1 virus after 1,2 and 3 days were assembled to produce 151,970 transcripts (Additional file 2: Table S1, Fig. 1). We used the above pipeline shown in Fig. 1 to identify duck lncRNAs. Among these assembled

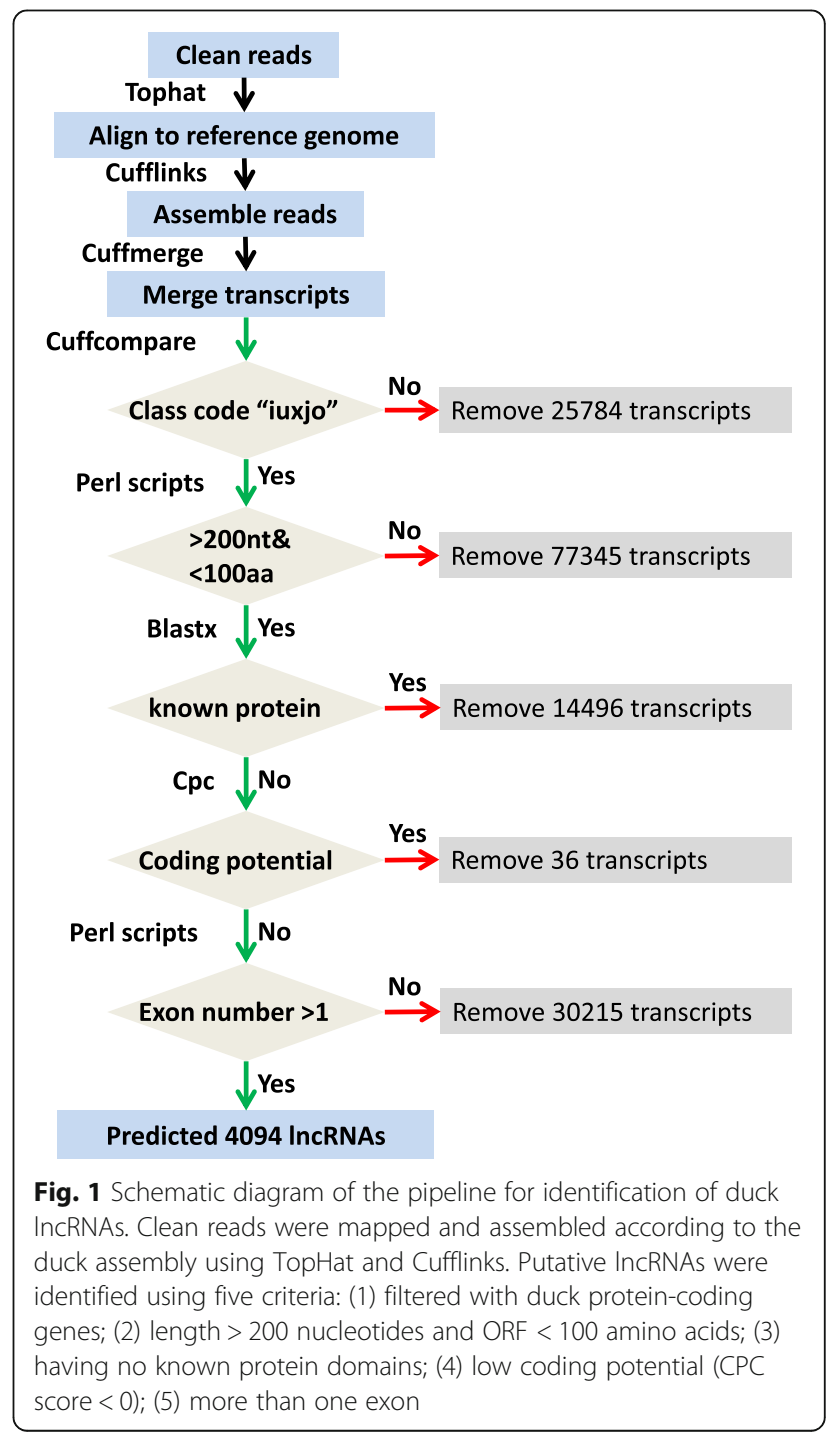


transcripts, 126,186 were novel or unknown transcripts and 48,841 were putative lncRNAs having a nucleotide length longer than $200 \mathrm{nt}$ and ORF shorter than 100 AA. We filtered 48,841 transcripts and found 9765 of them being homologous to duck and chicken protein. We further filtered the remained 39,076 transcripts and removed 3731 transcripts being homologous to protein-coding genes in the three protein databases (Swiss-Prot, KEGG and NR). After that, we assessed the protein-coding potential of 35,345 transcripts using the CPC program and deleted 36 potential coding transcripts [21]. Finally, we removed 31,215 transcripts that had only one exon. This effort finally identified 4094 transcripts from 3108 loci as putative multi-exons lncRNAs (Fig. 1 and Additional file 3). In order to identify the accuracy and quality of these annotated duck lncRNAs, we blasted them to the non-redundant database in the NCBI (RefSeq) and found 752 were annotated in this database.

\section{Characteristics of duck putative IncRNAs}

LncRNAs were classified into three types (intergenic, antisense and overlap lncRNAs) according to their locations relative to the nearest protein-coding genes. Among these 4094 duck lncRNAs, a large proportion $(87.6 \%)$ was located in the intergenic regions, $8.4 \%$ was antisense transcripts of protein-coding genes, while only $4.0 \%$ was overlapped lncRNAs (Fig. 2a). We further counted the distribution of transcript length, exon number and expression level of 4094 lncRNAs and compared them to 16,353 duck protein-coding transcripts. This effort found that 4094 duck lncRNA transcripts had an average length of $1903 \mathrm{nt}, 39.25 \%$ of them were 200 to $1000 \mathrm{nt}$ and $60.75 \%$ of them were larger than $1000 \mathrm{nt}$ in length (Fig. 2c). In contrast, the average length of duck mRNAs (1687 nt) was smaller than that of duck lncRNAs. For gene structure, duck lncRNAs contained two to nine exons with an average of 2.4 exons. This number is smaller than the average of duck mRNAs (average 10.2) (Fig. 2d). We further compared expressional patterns of lncRNAs and protein-coding genes in three tissues (brain, lung and spleen). The average expression levels of the putative lncRNA genes (brain average 15.7 FPKM, lung average 10.7 FPKM and spleen average 9.9 FPKM) tend to be lower than those of the protein-coding
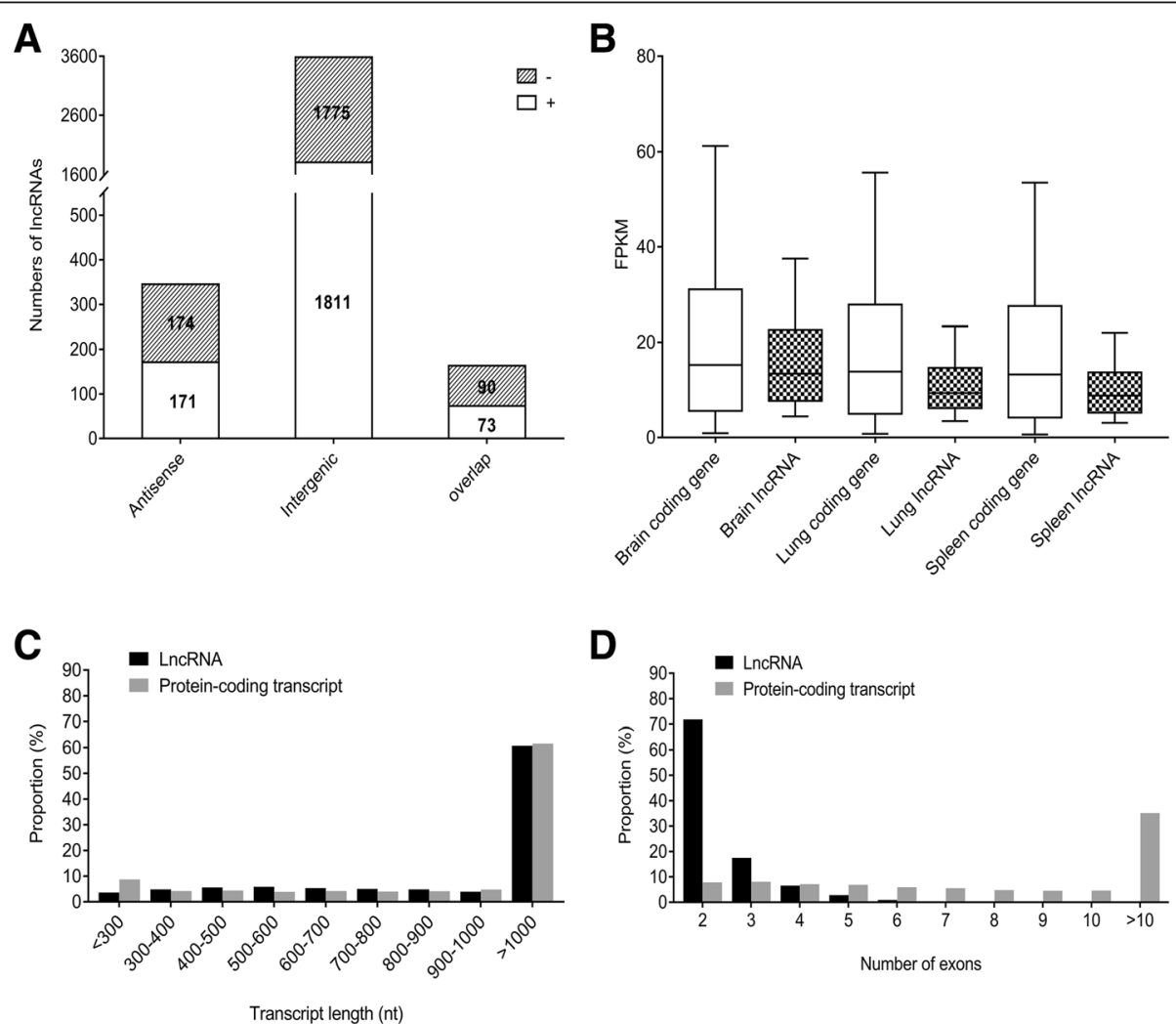

Fig. 2 Categories and features of the 4094 predicted duck IncRNAs. a Categories of duck IncRNAs divided based on genomic location between IncRNAs and protein-coding genes. "-" or "+" strand for each of the three main types was labeled on the columns. b-d Duck IncRNAs are longer, have fewer exons and have lower expression levels than protein-coding genes. Expression (b), distribution of length (c) and number of exons (d) of 4094 IncRNAs and 16,353 protein-coding transcripts of duck (BGl_duck_1.0) 
genes (brain average 19.8 FPKM, lung average 17.8 FPKM and spleen average 17.3 FPKM) in all three tissues (Fig. 2b).

\section{Identification of differentially expressed duck IncRNAs (DEIncRNAs)}

We compared lncRNA expression in duck infected with H5N1 viruses (DK/49-infected or GS/65-infected) against control individuals and identified a total of 619 DElncRNAs (FDR $\leq 0.05$, fold change $\geq 2$ ) including 323, 217 and 206 DElncRNAs in the brain, lung and spleen respectively (Fig. 3a and Additional file 4: Table S2). In lung, DK/49-infected ducks had 46, 71 and 51 lncRNAs with significantly changed expression 1-3 d after inoculation, and GS/65-infected ducks had 50, 37 and 48 lncRNAs with significantly changed expression 1-3 d after inoculation when compared to control ducks, while DK/49-infected ducks had 51, 54 and 47 lncRNAs showing significantly differential expression when compared to the corresponding GS/65-infected ducks 1-3 d after inoculation respectively (Fig. 3a and Additional file 4:
Table S2). Similar to lung tissue, DK/49- and GS/65-infected ducks showed similar number of (33 vs. 24, 35 vs. 48) DElncRNAs when compared to control individuals in brain and spleen in early infected time $(1 \mathrm{~d}$ after inoculation). Such differences in number of DElncRNAs was magnified in later infected time (2-3 d after inoculation), except that DK/49- and GS/65-infected ducks had similar number of DElncRNAs in brain in $2 \mathrm{~d}$ after inoculation (Fig. 3a). Detailed analysis indicated that, among 619 duck DElncRNAs, 29 were significantly differentially expressed in all three tissues (Fig. 3b). 172 lncRNAs were differentially expressed in both DK/49-infected and GS/65-infected ducks (Fig. 4c). These results suggested that the DElncRNAs might be involved into immune response to influenza A virus infection in ducks.

Prediction the target protein-coding gene of IncRNA in cis and in trans

LncRNAs inhibit or activate the transcription of genes located in their upstream or downstream by recruiting

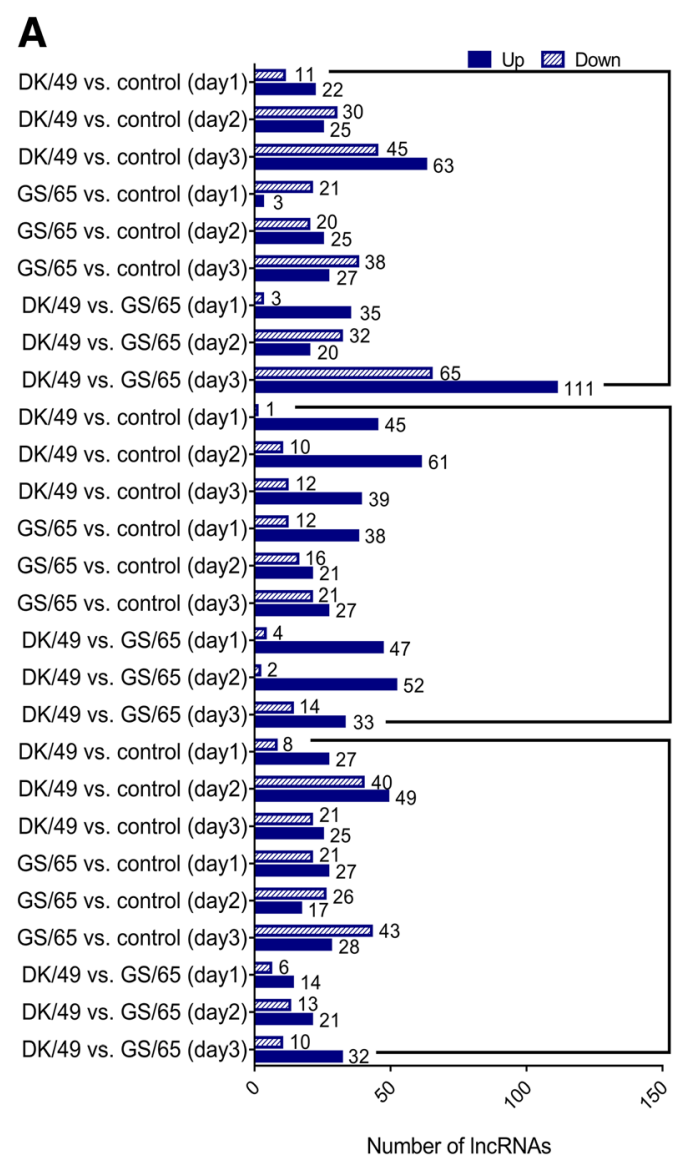

\section{B}

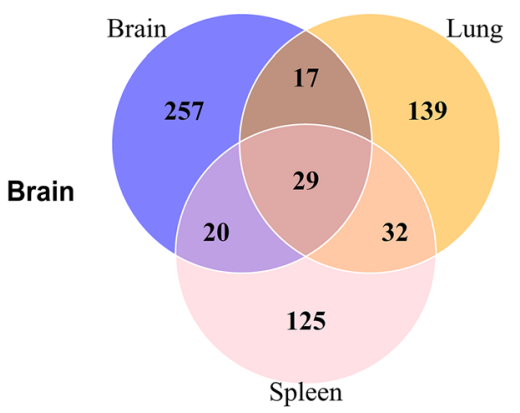

Lung

C

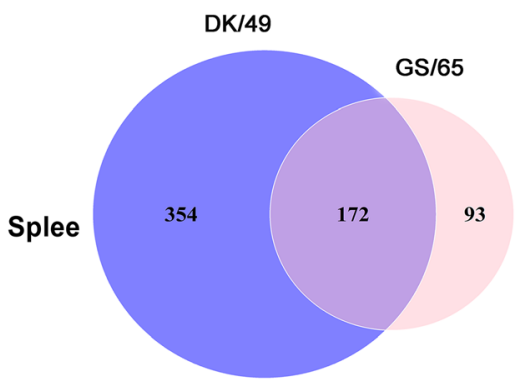

Fig. 3 DEIncRNAs between infected ducks and control ducks. a The numbers of DElncRNAs in three tissues. b Venn diagram showing nonoverlap and overlap among putative IncRNAs from brain, lung and spleen tissues. $\mathbf{c}$ Venn diagram showing non-overlap and overlap between putative IncRNAs from DK/49- and GS/65-infected ducks 


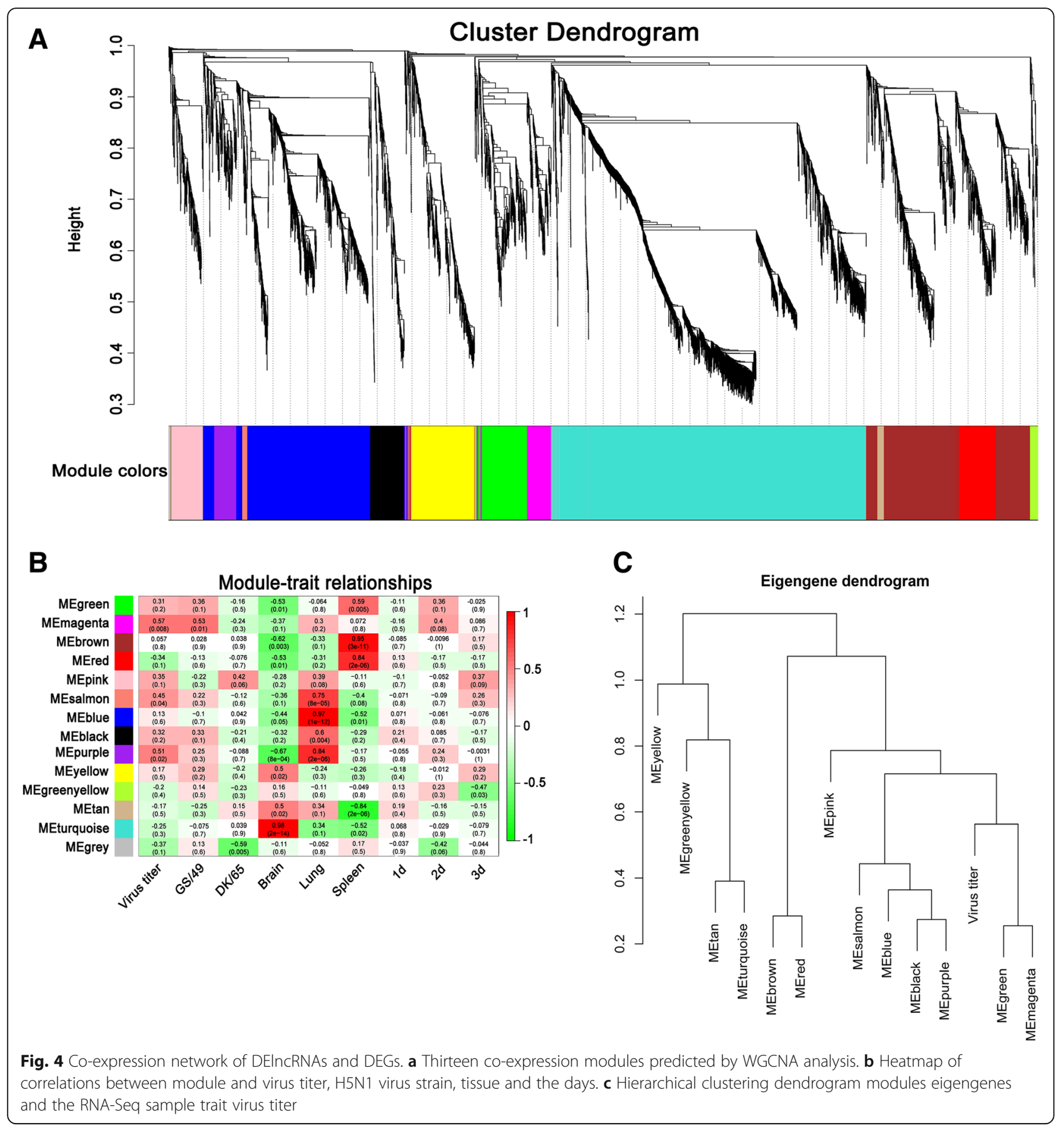

chromatin remodeling factors or protein complex [22]. These lncRNAs function in cis, acting on linked genes in the vicinity of the RNA's site. In order to identify duck lncRNAs functioning in cis, we predicted the potential protein-coding gene targets of lncRNAs among differentially expressed genes (DEGs) using $10 \mathrm{~kb}$ and $100 \mathrm{~kb}$ as the cutoff (Table 1). We detected 972/11,508 lncRNAs-coding genes pairs when used the $10 / 100 \mathrm{~kb}$ as the cutoff, and 721/106 DElncRNAs-DEGs pairs when used the $10 / 100 \mathrm{~kb}$ as the cutoff. We further found that
365 DElncRNAs were neighbored to 627 DEGs within $100 \mathrm{~kb}$, and 86 DElncRNAs were neighbored to 103 DEGs within $10 \mathrm{~kb}$ (Table 1 and Additional file 5: Table S3).

Moreover, lncRNAs act in trans, regulating genes far away from them or even in other chromosomes [23]. We predicted the targets of lncRNAs in trans-regulatory relationships using co-expression analysis [24]. The lncRNAs-coding genes co-expression networks were performed with 619 DElncRNAs and 5594 DEGs using 
Table 1 Summary of IncRNA-coding neighbor gene pairs

\begin{tabular}{lllllll}
\hline & IncRNAs & Coding genes & DElncRNAs & DEGs & IncRNA-coding gene pairs & DElncRNA-DEGs pairs \\
\hline $100 \mathrm{~kb}$ & 2632 & 7914 & 365 & 627 & 11,508 & 721 \\
$10 \mathrm{~kb}$ & 747 & 889 & 86 & 103 & 972 & 106
\end{tabular}

We searched for protein-coding genes $100 \mathrm{~kb} / 10 \mathrm{~kb}$ upstream and downstream of the identified IncRNAs. IncRNA-coding gene pairs/DElncRNA-DEGs pairs: the number of gene pairs of IncRNAs (or DEIncRNAs) and protein-coding genes (or DEGs) by using $100 \mathrm{~kb} / 10 \mathrm{~kb}$ as cutoff; $100 \mathrm{~kb} / 10 \mathrm{~kb}$ : the number of IncRNAs (or DElncRNAs) and protein-coding genes (or DEGs) by using $100 \mathrm{~kb} / 10 \mathrm{~kb}$ as cutoff

the WGCNA package (Additional file 4: Table S2, Additional file 6: Table S4 and Additional file 7: Table S5). As a consequence, 13 co-expression modules were constructed in size from 2261 genes in the turquoise module to 36 genes in the salmon module (Fig. 4a and Additional file 8: Table S6).

Functional analysis indicated that 5594 expressed DEGs were enriched in $640 \mathrm{GO}$ terms (401 GO under biological process, 144 GO under cellular component and 95 GO under molecular function) (Additional file 9: Table S7). The results showed that DEGs from magenta and green modules were associated with immune response in the biological process, including innate immune response (GO: 0045087), defense response to virus (GO: 0051607), positive regulation of defense response to virus by host (GO: 0002230), immune response (GO: 0006955) and negative regulation of viral genome replication (GO: 0045071).

Among 13 co-expression modules, magenta module had the highest correlation with virus titer (Fig. 4b, c), which included 16 DElncRNAs and 174 DEGs. Detailed analysis indicated that both the magenta and green modules contained many known anti-influenza A virus immune genes (such as $N F-\kappa B, F A D D$, IFNA, IFNG, IRF7, IL8, IFNK, OASL, AVIFIT, MDA5 and TRIM25)

(Fig. 5), supporting their critical role in host response to influenza A virus infection. Such inference was further supported by pathway analysis (KEGG), which demonstrated that DEGs in the magenta and green modules were significantly enriched $(p<0.05)$ in nine pathways including Influenza $\mathrm{A}$, Herpes simplex infection, RIG-I-like receptor signaling pathway, Cytokine-cytokine receptor interaction, Toll-like receptor signaling pathway, NOD-like receptor signaling pathway, Intestinal immune network for IgA production, Arginine biosynthesis, Alanine, aspartate and glutamate metabolism, and Cytosolic DNA-sensing pathway (Additional file 10: Table S8).

\section{Real-time quantitative PCR (qPCR) validation}

Expression of eight DElncRNAs from the magenta or green modules in DEF cells before or after infected by H5N1 virus (12 h, $24 \mathrm{~h}$ and $36 \mathrm{~h}$ ) were examined using qRT-PCR (Fig. 6). This analysis indicated that, similar to duck in vivo transcriptomic expressional profiles, all tested eight IncRNAs were significantly increased $(p<$

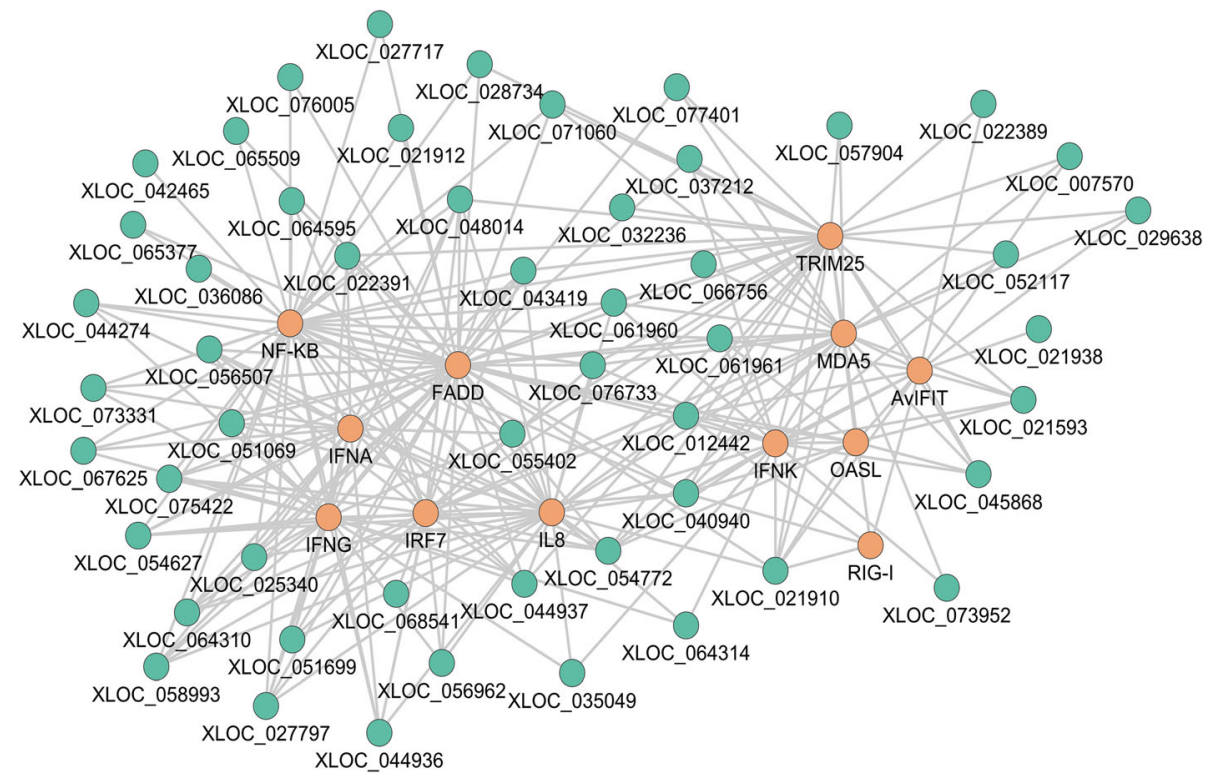

Fig. 5 The magenta and green co-expressional networks of DEIncRNAs and DEGs. Orange nodes represent IncRNAs, green nodes represent coding genes. The line between IncRNAs and coding genes indicates an expression pattern correlation 

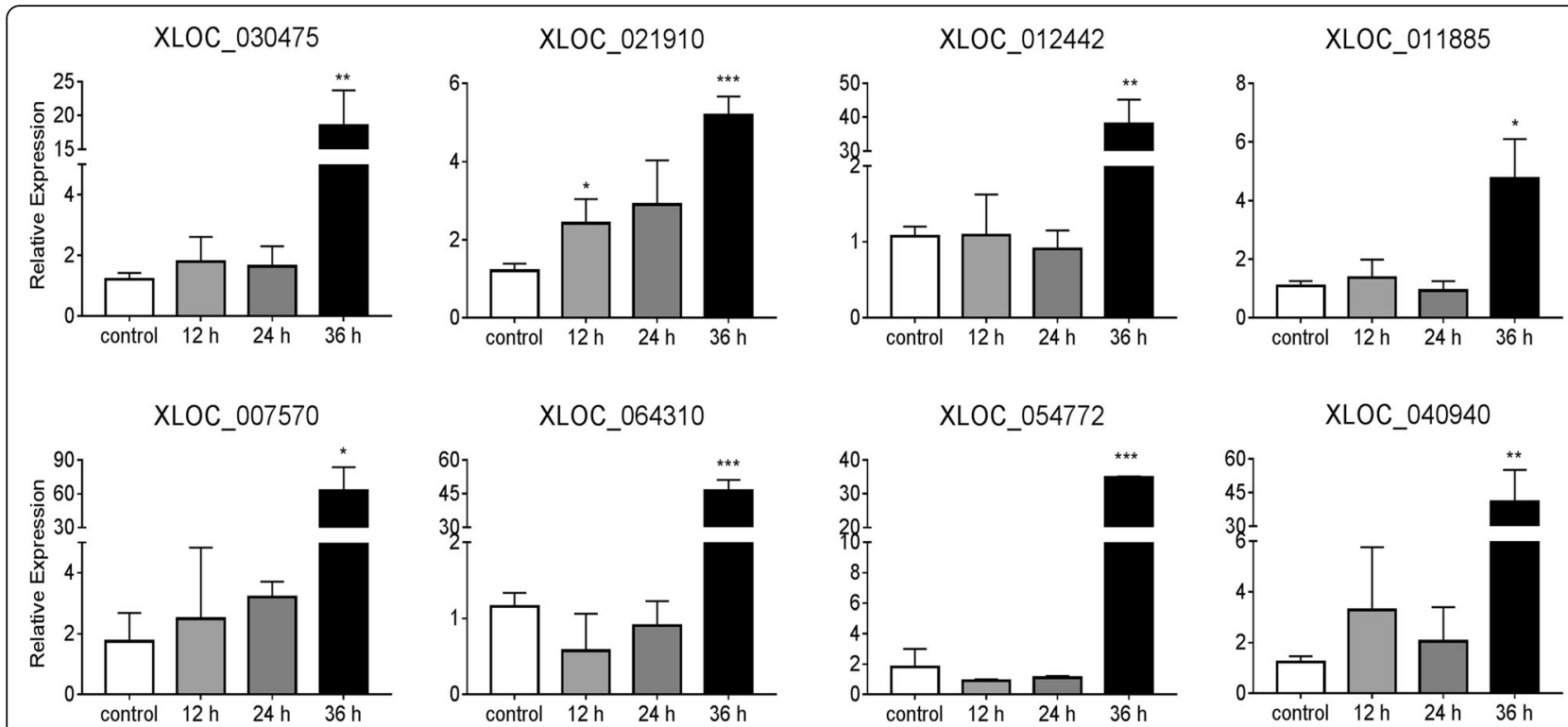

Fig. 6 Expression of eight DEIncRNAs from magenta and green modules in DEF cells before and after infected with H5N1 virus. Expression in cells were counted relative IncRNA level to that of reference gene-GAPDH and presented as fold change versus the corresponding of DEF control cells (without H5N1 virus infection) (two-tailed Student's test, $n=3$ ). The data are expressed as the mean \pm SD. ${ }^{*} P<0.05$, ${ }^{* *} P<0.01,{ }^{* *} P<0.001$

0.05 ) in DEF cells infected by CK/0513 (A chicken/ hubei/0513/2007) H5N1 virus after 36 h. Such consistent expressional distribution of these DElncRNAs in vivo and in vitro was further supported that they might play important roles in host immune response to influenza A virus. Our ability to assess the function of these eight DElncRNAs using genetic manipulations will certainly extend knowledge of lncRNA related to influenza in ducks.

\section{Discussion}

Accumulated studies demonstrated that lncRNAs extensively involved in various biological processes in animals, including embryogenesis [25, 26], muscle development $[27,28]$, adipogenesis [29] and immune responses [15, 30]. Therefore, it is interested to annotate and identify candidate lncRNA related to diseases, such as influenza, using transcriptomic data. In the present study, we built a screening platform to identify lncRNAs after explored features of 62,447 lncRNAs from five model animals (Fig. 1). This pipeline removed known protein-coding genes using the most effective computational methods available to date, such as Cufflinks and CPC. Unlike the previously reserved intergenic transcripts [31], we retained the intergenic transcripts, antisense transcripts and transcripts that overlap with the protein-coding genes. We removed the transcripts having ORF length more than 100 AA. In addition, we filtered the potential protein-coding genes through blasting with duck protein sequences, chicken protein sequences and other sequences from protein databases (KEGG, Swiss-Prot and NR data sets). This extended range of comparison could remove the transcripts that have encoded potential. Finally, we retained the multi-exons transcripts as predicted lncRNAs according to the characteristics of model animals' lncRNAs.

LncRNAs are a group of endogenous RNAs that function as regulators of gene expression. We detected 619 putative DElncRNAs among control ducks and individuals infected with $\mathrm{H} 5 \mathrm{~N} 1$ virus (Fig. 3). We predicted function of DElncRNAs in cis and trans. The cis lncRNAs act on the neighboring genes. In trans, gene executes cellular processes in functional modules [32]. Therefore, this study constructed the weighted gene co-expression network and identified modules related to H5N1 virus titer. Among 13 detected modules (Fig. 4b), the magenta module was correlated with virus titer and DK/49 virus, while the other modules were correlated with GS/65 or tissues or the days of duck growth and development after inoculated. Moreover, we found that the green module was highly associated with magenta module and virus titer. Interestingly, TRIM25, IFNK, DDX58 (RIG-I), AvIFIT and OASL were found to be a highly connected gene in the magenta module, and IL8, $F A D D, N F-K B, I R F 7, I F N E, I F N A$ and CXCL6 in the green module, these protein-coding genes play important roles in anti-virus immune response (Fig. 5). For example, Jiang et al. [33] found an IFN-induced long noncoding RNA lnc-Lsm3b, played a negative feedback regulatory role in the late immune response and 
inhibited the activity of RIG-I. Lnc-Lsm3b competes to bind RIG-I monomers with viral RNAs using its multivalent long stem domain, prevents conformational changes of RIG-I and activation of its downstream signaling. Here, we found that RIG-I was co-expressed with XLOC_021920, XLOC_012442 and XLOC_040940 (weight $>=0.2$ ) in the magenta and green modules. These three lncRNAs showed differentially expressed in all three $\mathrm{H} 5 \mathrm{~N} 1$ infections in vivo and in vitro, implying that might affect function of RIG-I in the immune response influenza A viruses and be interested to further functional studies.

Due to limitations of available duck transcriptomes, annotations of lncRNAs in this study are not comprehensive. These include: (1) some duck lncRNAs lacking polyadenylation might be not annotated using mRNA transcriptome; (2) due to the inherent limitations of using 90 bp paired-end RNA-Seq, we did not get full sequences of all annotated duck lncRNAs; (3) some lncRNAs may be annotated incorrectly due to error of the available duck assembly.

\section{Conclusion}

LncRNAs have been functionally annotated and studied extensively in mammals. However knowledge even sequences of avian lncRNA, especially duck lncRNA, is scarce. We first identified duck lncRNAs associated with immune response to avian influenza virus using RNA-Seq data. We present a pipeline to investigate the duck lncRNAs and predicted the function of lncRNAs based on the coding gene neighbor loci and the enriched functions of co-expression protein-coding genes. These analyses together with duck lncRNA sequences provide information to understand their functions in the immune response.

\section{Methods}

\section{Paired-end RNA sequencing}

The RNA-Seq data (SRA accession SRP052742) were from the duck genome paper [14].

\section{Reads mapping and assembly}

After clean reads, we build the index of the reference genome [14] using Bowtie v2.2.6 and aligned paired-end clean reads to the reference genome using Tophat v2.1.0. Tophat was run with "-N 3 --read-edit-dist 5 -r 20 -a 9 -i 30 -I 4000 --min-segment-intron 30 -max-segment-intron 4000 --min-coverage-intron 30 --max-coverage-intron 4000 --microexon-search" and "--phred64-quals", other parameters were set as default. Only the mapped reads with number of mismatches less than three were used to construct transcripts using Cufflinks (v2.2.1) in a reference-based approach. Finally, we used Cuffmerge to merge all the transcripts into a final irredundant transcript GTF file.
Describing feature of IncRNAs and protein-coding genes A total of 4094 lncRNAs and 16,353 mRNAs were characterized in transcript length, exon number and expressional profiles. Transcript length categories were $<300$, 300-400, 400-500, 500-600, 600-700, 700-800, 800900, 900-1000, and $>1000$ nucleotides. Exon number categories were: $1,2,3,4,5,6,7,8,9,10$, and $>10$. The proportion of different types of lncRNAs and protein-coding transcripts were calculated. Furthermore, we used brain, lung and spleen transcriptomes of control ducks to characterize the expression pattern of the lncRNA genes and coding genes, whose FPKM value were calculated using the Cufflinks (v2.2.1) program.

\section{Identifying differentially expressed IncRNAs and neighbor target genes}

Differentially expressed lncRNAs between control ducks and ducks infected by $\mathrm{H} 5 \mathrm{~N} 1$ viruses were identified using the Cuffdiff program with the thresholds of $\mid$ fold change $\mid \geq$ 1 and adjusted $p$-values $<0.05$. Protein-coding genes were within $10 \mathrm{~kb} / 100 \mathrm{~kb}$ upstream and downstream of the identified lncRNAs were inferred as neighbor target genes.

\section{Analysis of co-expression, GO enrichment and KEGG pathway}

Co-expression networks were built using the WGCNA package [24]. First, we used FPKM value of DEGs and DElncRNAs as the input file (Additional file 7: Table S5). We then chose "soft power $=8$ " using the scale-free topology criterion. After that, we constructed networks using the blockwiseModules function in the WGCNA package with the minimum module size to 30 genes, and the minimum height for merging modules at 0.15 (default value). For each module, we calculated module membership (also known as module eigengene based connectivity $\mathrm{k}_{\mathrm{ME}}$ ) based on Pearson's correlation [24]. Finally, we used the virus titers of three tissues as trait data to calculate the GS value (gene Trait Significance). We chose modules which had a highly strength connection with virus titers. To visualize the chose modules (together with GO and KEGG analysis), the connections between the IncRNAs and the mRNAs were shown using the Cytoscape program [34].

GO category and KEGG enrichment were performed using DAVID software [35] and KOBAS software [36] using a threshold of $P<0.05$, respectively.

\section{Cell culture, RNA isolation and qRT-PCR}

Duck embryonic fibroblast (DEF) cells were isolated from eleven-day-old embryos. Duck embryos were digested with trypsin, suspended and filtered using gauze. DEF cells were diluted and cultured in six-well culture plates in an atmosphere of $5 \% \mathrm{CO}_{2}$ at $37^{\circ} \mathrm{C}$. Cells with a coverage $\sim 90 \%$ were collected or infected 
with $\mathrm{H} 5 \mathrm{~N} 1$ virus. $\mathrm{H} 5 \mathrm{~N} 1$ virus infected cells were collected after infection at 12,24 and $36 \mathrm{~h}$, respectively.

Total RNA was isolated from cells using RNeasy ${ }^{\circ}$ Mini Kit (QIAGEN, Germany). RNA quality was measured using a NanoDrop-2000 spectrophotometer (Thermo Fisher Scientific, USA) and by agarose gel electrophoresis. Genomic DNA was removed from extracted total RNA using DNase treatment. A $2 \mu \mathrm{g}$ aliquot of total RNA was used for cDNA synthesis using a M-MLV Reverse Transcriptase (Promega, USA) with Oligo (dT) $)_{15}$ Primer.

Quantitative RT-PCR was performed using SYBR Green PCR Master Mix with a real-time PCR System ABI7500 (ABI, USA). Quantitative RT-PCR condition were as follows: $94^{\circ} \mathrm{C}$ for $2 \mathrm{~min}$, followed by 40 cycles of $95^{\circ} \mathrm{C}$ for $5 \mathrm{~s}$ and $60^{\circ} \mathrm{C}$ for 30s. Fluorescence changes of SYBR Green were monitored automatically in each cycle, and the threshold cycle $(\mathrm{Ct})$ over the background was calculated for each reaction. Samples with three biological replicates were normalized using GAPDH, and the relative expression levels were measured using the $2^{-\triangle \Delta C t}$ analysis method. Student's $t$-test was used to determine whether the qRT-PCR results were statistically different from two samples $\left({ }^{*} P<0.05 ;{ }^{* *} P<0.01\right)$. PCR primers are listed in Additional file 11: Table S9.

\section{Additional files}

Additional file 1: Figure S1. Features of IncRNA in five organisms. Length distribution of duck IncRNAs transcript (A), ORF length (B) and numbers of exons (C) were shown in the picture. (TIF $3391 \mathrm{~kb}$ )

Additional file 2: Table S1. Summary of RNA-Seq data and reads mapped to the Anas platyrhynchos genome. (DOCX $21 \mathrm{~kb}$ )

Additional file 3: Information of IncRNA annotated with GTF files. (GTF 1785 kb)

Additional file 4: Table S2. DElncRNAs detected between duck infected with DK/49 or GS/65 H5N1 virus and control individuals. (XLSX $176 \mathrm{~kb})$

Additional file 5: Table S3. Protein-coding genes located within 10/ $100 \mathrm{~kb}$ upstream and downstream of duck IncRNAs. (XLSX 642 kb)

Additional file 6: Table S4. List of DEGs used in co-expression analysis. (XLSX $2055 \mathrm{~kb}$ )

Additional file 7: Table S5. FPKM value of DElncRNAs and DEGs. (CSV $1273 \mathrm{~kb}$ )

Additional file 8: Table S6. Thirteen co-expression modules were predicted with the WGCNA package. (CSV 2346 kb)

Additional file 9: Table S7. GO enrichment analysis of DEGs coexpressed with DEIncRNAs. (XLSX $135 \mathrm{~kb})$

Additional file 10: Table S8. KEGG analysis of DEGs co-expressed with DEIncRNAs. (XLSX $83 \mathrm{~kb}$ )

Additional file 11: Table S9. Primers used in qRT-PCR analysis. (DOCX $18 \mathrm{~kb}$ )

\section{Abbreviations}

DEGs: Differentially expressed genes; DElncRNAs: Differentially expressed IncRNAs; DK/49: A highly pathogenic (A/duck/Hubei/49/05) H5N1 virus; GO: Gene ontology; GS/65: A weakly pathogenic (A/goose/Hubei/65/05) H5N1 virus; KEGG: Kyoto Encyclopedia of Genes and Genomes; LncRNAs: Long non-coding RNAs; ORF: Open reading frame

\section{Acknowledgements}

We would like to thank Dr. Benzhong Zhu (China Agricultural University) for technical assistance of bioinformatics analysis.

\section{Funding}

This study was funded by the National Natural Science Foundation of China (31471176 and 31772587)

\section{Availability of data and materials}

The RNA-Seq data were downloaded from the National Center for Biotechnology Information (NCBI) Sequence Read Archive (SRA) database under the SRA accession SRP052742.

\section{Authors' contributions}

$\mathrm{YHH}$ and $\mathrm{JHL}$ designed this project. CL performed transcriptomic analysis and constructed gene co-expression network. YLX performed influenza A virus infection and replication experiments. HC performed quantitative RTPCR experiments. YRS aligned the IncRNAs to RefSeq data. CL wrote the manuscript. $\mathrm{YHH}, \mathrm{CL}$ and $\mathrm{YRS}$ revised the manuscript. All authors read and approved the final manuscript.

Ethics approval and consent to participate

Not applicable.

\section{Consent for publication}

Not applicable.

\section{Competing interests}

The authors declare that they have no competing interests.

\section{Publisher's Note}

Springer Nature remains neutral with regard to jurisdictional claims in published maps and institutional affiliations.

\section{Author details}

'State Key Laboratory of Agrobiotechnology, China Agricultural University, Beijing 100193, China. ${ }^{2}$ Key Laboratory of Animal Epidemiology and Zoonosis, Ministry of Agriculture, College of Veterinary Medicine, China Agricultural University, Beijing, China.

Received: 12 June 2018 Accepted: 27 December 2018

Published online: 11 January 2019

\section{References}

1. Djebali S, Davis CA, Merkel A, Dobin A, Lassmann T, Mortazavi A, Tanzer A, Lagarde J, Lin W, Schlesinger F, et al. Landscape of transcription in human cells. Nature. 2012;489(7414):101-8.

2. Mudge JM, Harrow J. Creating reference gene annotation for the mouse C57BL6/J genome assembly. Mamm Genome. 2015;26(9-10):366-78.

3. Birney E, Stamatoyannopoulos JA, Dutta A, Guigo R, Gingeras TR, Margulies EH, Weng Z, Snyder M, Dermitzakis ET, Thurman RE, et al. Identification and analysis of functional elements in 1\% of the human genome by the ENCODE pilot project. Nature. 2007:447(7146):799-816.

4. Ulitsky I. Evolution to the rescue: using comparative genomics to understand long non-coding RNAs. Nat Rev Genet. 2016:17(10):601-14.

5. Nelson BR, Makarewich CA, Anderson DM, Winders BR, Troupes CD, Wu F, Reese AL, McAnally JR, Chen X, Kavalali ET, et al. A peptide encoded by a transcript annotated as long noncoding RNA enhances SERCA activity in muscle. Science. 2016:351(6270):271-5.

6. Matsumoto A, Pasut A, Matsumoto M, Yamashita R, Fung J, Monteleone E, Saghatelian A, Nakayama KI, Clohessy JG, Pandolfi PP. mTORC1 and muscle regeneration are regulated by the LINC00961-encoded SPAR polypeptide. Nature. 2017;541(7636):228-32

7. Kino T, Hurt DE, Ichijo T, Nader N, Chrousos GP. Noncoding RNA gas5 is a growth arrest- and starvation-associated repressor of the glucocorticoid receptor. Sci Signal. 2010;3(107):ra8.

8. Hung T, Wang Y, Lin MF, Koegel AK, Kotake $Y$, Grant GD, Horlings HM, Shah $\mathrm{N}$, Umbricht C, Wang $\mathrm{P}$, et al. Extensive and coordinated transcription of noncoding RNAs within cell-cycle promoters. Nat Genet. 2011;43(7):621-9.

9. Anderson DM, Anderson KM, Chang CL, Makarewich CA, Nelson BR, McAnally JR, Kasaragod P, Shelton JM, Liou J, Bassel-Duby R, et al. A 
micropeptide encoded by a putative long noncoding RNA regulates muscle performance. Cell. 2015;160(4):595-606.

10. Wu Y, Wu Y, Tefsen B, Shi Y, Gao GF. Bat-derived influenza-like viruses H17N10 and H18N11. Trends Microbiol. 2014;22(4):183-91.

11. Munster VJ, Baas C, Lexmond P, Waldenstrom J, Wallensten A, Fransson T, Rimmelzwaan GF, Beyer WE, Schutten M, Olsen B, et al. Spatial, temporal, and species variation in prevalence of influenza a viruses in wild migratory birds. PLoS Pathog. 2007;3(5):e61.

12. Jeong OM, Kim MC, Kim MJ, Kang HM, Kim HR, Kim YJ, Joh SJ, Kwon JH, Lee YJ. Experimental infection of chickens, ducks and quails with the highly pathogenic H5N1 avian influenza virus. J Vet Sci. 2009;10(1):53-60.

13. Barber MR, Aldridge JR Jr, Webster RG, Magor KE. Association of RIG-I with innate immunity of ducks to influenza. Proc Natl Acad Sci U S A. 2010; 107(13):5913-8.

14. Huang Y, Li Y, Burt DW, Chen H, Zhang Y, Qian W, Kim H, Gan S, Zhao Y, Li $J$, et al. The duck genome and transcriptome provide insight into an avian influenza virus reservoir species. Nat Genet. 2013;45(7):776-83.

15. Ouyang J, Zhu X, Chen Y, Wei H, Chen Q, Chi X, Qi B, Zhang L, Zhao Y, Gao $G$, et al. NRAV, a long noncoding RNA, modulates antiviral responses through suppression of interferon-stimulated gene transcription. Cell Host Microbe. 2014;16(5):616-26.

16. Winterling C, Koch M, Koeppel M, Garcia-Alcalde F, Karlas A, Meyer TF. Evidence for a crucial role of a host non-coding RNA in influenza a virus replication. RNA Biol. 2014;11(1):66-75.

17. Li T, Wang S, Wu R, Zhou X, Zhu D, Zhang Y. Identification of long nonprotein-coding RNAs in chicken skeletal muscle using next generation sequencing. Genomics. 2012;99(5):292-8.

18. Chen CK, Yu CP, Li SC, Wu SM, Lu MJ, Chen YH, Chen DR, Ng CS, Ting CT, Li WH. Identification and evolutionary analysis of long non-coding RNAs in zebra finch. BMC Genomics. 2017;18(1):117.

19. Okazaki Y, Furuno M, Kasukawa T, Adachi J, Bono H, Kondo S, Nikaido I, Osato N, Saito R, Suzuki H, et al. Analysis of the mouse transcriptome based on functional annotation of 60,770 full-length cDNAs. Nature. 2002; 420(6915):563-73.

20. Trapnell C, Roberts A, Goff L, Pertea G, Kim D, Kelley DR, Pimentel H, Salzberg SL, Rinn JL, Pachter L. Differential gene and transcript expression analysis of RNA-Seq experiments with TopHat and cufflinks. Nat Protoc. 2012;7(3):562-78.

21. Kong L, Zhang Y, Ye ZQ, Liu XQ, Zhao SQ, Wei L, Gao G. CPC: assess the protein-coding potential of transcripts using sequence features and support vector machine. Nucleic Acids Res. 2007;35(Web Server issue):W345-9.

22. McHugh CA, Chen CK, Chow A, Surka CF, Tran C, McDonel P, Pandya-Jones A, Blanco M, Burghard C, Moradian A, et al. The Xist IncRNA interacts directly with SHARP to silence transcription through HDAC3. Nature. 2015; 521(7551):232-6

23. Rinn JL, Kertesz M, Wang JK, Squazzo SL, Xu X, Brugmann SA, Goodnough $\mathrm{LH}$, Helms JA, Farnham PJ, Segal E, et al. Functional demarcation of active and silent chromatin domains in human HOX loci by noncoding RNAs. Cell. 2007;129(7):1311-23

24. Langfelder P, Horvath S. WGCNA: an R package for weighted correlation network analysis. BMC Bioinf. 2008:9:559,

25. Zhao J, Sun BK, Erwin JA, Song JJ, Lee JT. Polycomb proteins targeted by a short repeat RNA to the mouse X chromosome. Science. 2008;322(5902): 750-6.

26. Caballero J, Gilbert I, Fournier E, Gagne D, Scantland S, Macaulay A, Robert C. Exploring the function of long non-coding RNA in the development of bovine early embryos. Reprod Fertil Dev. 2014;27(1):40-52.

27. Zhan S, Dong Y, Zhao W, Guo J, Zhong T, Wang L, Li L, Zhang H. Genomewide identification and characterization of long non-coding RNAs in developmental skeletal muscle of fetal goat. BMC Genomics. 2016;17:666.

28. Sun X, Li M, Sun Y, Cai H, Lan X, Huang Y, Bai Y, Qi X, Chen H. The developmental transcriptome sequencing of bovine skeletal muscle reveals a long noncoding RNA, IncMD, promotes muscle differentiation by sponging miR-125b. Biochim Biophys Acta. 2016;1863(11):2835-45.

29. Zhang T, Zhang X, Han K, Zhang G, Wang J, Xie K, Xue Q. Genome-Wide Analysis of IncRNA and mRNA Expression During Differentiation of Abdominal Preadipocytes in the Chicken. G3 (Bethesda). 2017;7(3):953-66.

30. Atianand MK, Hu W, Satpathy AT, Shen Y, Ricci EP, Alvarez-Dominguez JR, Bhatta A, Schattgen SA, McGowan JD, Blin J, et al. A long noncoding RNA lincRNA-EPS acts as a transcriptional brake to restrain inflammation. Cell. 2016;165(7):1672-85.
31. Luo H, Bu D, Sun L, Fang S, Liu Z, Zhao Y. Identification and function annotation of long intervening noncoding RNAs. Brief Bioinform. 2017;18(5): 789-97.

32. Carlson MR, Zhang B, Fang Z, Mischel PS, Horvath S, Nelson SF. Gene connectivity, function, and sequence conservation: predictions from modular yeast co-expression networks. BMC Genomics. 2006;7:40.

33. Jiang M, Zhang S, Yang Z, Lin H, Zhu J, Liu L, Wang W, Liu S, Liu W, Ma Y, et al. Self-recognition of an inducible host IncRNA by RIG-I feedback restricts innate immune response. Cell. 2018;173(4):906-19 e913.

34. Shannon P, Markiel A, Ozier O, Baliga NS, Wang JT, Ramage D, Amin N, Schwikowski B, Ideker T. Cytoscape: a software environment for integrated models of biomolecular interaction networks. Genome Res. 2003;13(11): 2498-504.

35. Huang DW, Sherman BT, Lempicki RA. Systematic and integrative analysis of large gene lists using DAVID bioinformatics resources. Nat Protoc. 2009:4(1): 44-57.

36. Xie C, Mao XZ, Huang JJ, Ding Y, Wu JM, Dong S, Kong L, Gao G, Li CY, Wei LP. KOBAS 2.0: a web server for annotation and identification of enriched pathways and diseases. Nucleic Acids Res. 2011;39:W316-22.

Ready to submit your research? Choose BMC and benefit from:

- fast, convenient online submission

- thorough peer review by experienced researchers in your field

- rapid publication on acceptance

- support for research data, including large and complex data types

- gold Open Access which fosters wider collaboration and increased citations

- maximum visibility for your research: over $100 \mathrm{M}$ website views per year

At BMC, research is always in progress.

Learn more biomedcentral.com/submissions 